\title{
Short-Term Outcomes in Heart Transplant Recipients Treated with Generic Tacrolimus Compared to Prograf*
}

\author{
Vinayak Dhungel, Monica M. Colvin-Adams, Peter M. Eckman ${ }^{\#}$ \\ Department of Medicine, Cardiovascular Division, University of Minnesota Medical School, Minneapolis, USA \\ Email: \#eckmanp@umn.edu
}

Received May 22, 2012; revised June 30, 2012; accepted July 10, 2012

Copyright (C) 2013 Vinayak Dhungel et al. This is an open access article distributed under the Creative Commons Attribution License, which permits unrestricted use, distribution, and reproduction in any medium, provided the original work is properly cited.

\begin{abstract}
Calcineurin inhibitors are the cornerstone of solid organ transplant immunosuppression. Tacrolimus has only recently become available in a non-branded formulation, and there is little data on the efficacy and safety of generic tacrolimus, particularly following heart transplant. We performed a retrospective analysis of 21 consecutive patients who were treated with generic tacrolimus following heart transplant and compared rate of biopsy-proven acute cellular rejection to historical controls who were treated with Prograf. No significant difference in biopsy-proven acute cellular rejection was noted between the groups and rates of opportunistic infection and death were comparable. Although limited by the single-center, retrospective design, this preliminary data may be useful to clinicians facing the option of initiating generic tacrolimus following heart transplant.
\end{abstract}

Keywords: Generic Tacrolimus; Heart Transplant

\section{Background}

Calcineurin inhibitors (CNIs) are the cornerstone of solid organ transplant immunosuppression. Generic cyclosporin A has been available since mid-1980s as an antirejection medication, but Prograf (tacrolimus) has more recently become the dominant CNI [1] due to improved side effect profile and less nephrotoxicity. The Food and Drug Administration initially approved tacrolimus for livertransplant patients in the mid-1990s; since then, its usage has gradually extended to other allograft transplant recipients, including heart transplant recipients. Generic tacrolimus is now available and patients, pharmacies, and payers have a strong interest in minimizing post-transplant expenses. Transplant programs have expressed concern about the bioequivalence, efficacy, and safety of generic tacrolimus compared to Prograf [2-4], and the decision of whether to use generic tacrolimus is presently center-specific. In late 2009, we elected to permit hearttransplant patients to begin their de novo calcineurin inhibitor with generic tacrolimus rather than Prograf, as the frequent monitoring in the early post-transplant period was thought to provide the largest margin of safety. In

\footnotetext{
*Disclosure statement: We do not have any conflicts of interest relevant to this publication.

\#Corresponding author.
}

this report, we present our preliminary experience.

\section{Methods}

Retrospective analysis was performed on the first 21 consecutive patients treated with tacrolimus following heart transplant. Forty-four consecutive previous heart transplant patients who were treated with Prograf were studied as a comparison group. Adjunct immunosuppressants (IMS) in all patients consisted of mycophenolatemofetil (MMF) and steroids, which were typically discontinued within 6 months. Induction is not typically administered at our center, but IL-2 receptor antagonists (daclizumab or basiliximab) were given in select patients to permit delayed initiation of calcineurin inhibitors in the setting of marginal pre-transplant renal function. Tacrolimus levels at 1,3 and 6 months following heart transplant were considered for calculating the mean trough levels in both the groups. Rejection, infection, and graft function were evaluated 6 months after transplant. Continuous measurements were summarized using the mean and standard deviation. Counts and percentages were used to represent categorical variables. A $p$ value of $<0.05$ was considered to be statistically significant. Two-tailed t-test was used for comparison of continuous variables, and Fischer's exact or chi-square tests were 
used to compare categorical data. Biopsy results were tallied and rejection rate per patient-day over the 6month study period was calculated for each group to adjust for slight variations in biopsy frequency.

\section{Results}

Baseline characteristics are shown in Table 1; there were two significant differences between the cohorts. Three patients in the tacrolimus group had infected ventricular assist device (VAD) compared to 0 in the Prograf group ( $p=0.03$ ), and 4 patients in the Prograf group were allosensitized with panel reactive antibodies (PRA) $>20 \%$, compared to 2 in the tacrolimus group ( $p=$ NS). Following transplant, there was no significant difference in mean tacrolimus trough level, post-transplant diabetes, CMV (cytomegalovirus) or other opportunistic infection (Table 2). There was no significant difference in the IMS protocol or mean tacrolimus trough level across the two groups during the period studied. The opportunistic infections in the Prograf group included coagulase-negative Staphylococcal bacteremia, sternal wound Candidiasis, Staphylococcal sternal wound infection and Pseudomonas knee abscess. Opportunistic infections in the tacrolimus group were aspergillosis and HSV-2 viremia. Mean cardiac index was measured by Fick method during baseline testing in all patients at 1 month after transplant, and was not significantly different ( $p=0.17)$.

No significant difference in biopsy-proven acute cel-

Table 1. Baseline characteristics.

\begin{tabular}{|c|c|c|c|}
\hline & Tacrolimus $(\mathrm{n}=21)$ & Prograf $(n=44)$ & $p$-value \\
\hline Male & $10(48 \%)$ & 31 (70\%) & \\
\hline Mean age (years) & $56.8 \pm 10.2$ & $50.9 \pm 16.5$ & \\
\hline Hypertension & $18(85.7 \%)$ & $42(95.5 \%)$ & 0.17 \\
\hline History of ischemic heart failure & $14(66.6 \%)$ & $21(47.7 \%)$ & 0.16 \\
\hline Pre-transplant diabetes & $14(66.6)$ & $24(54.5 \%)$ & 0.36 \\
\hline VAD & $13(62 \%)$ & $22(50 \%)$ & 0.5 \\
\hline Infected VAD & $3(14 \%)$ & 0 & 0.03 \\
\hline Thymoglobulin & $4(19 \%)$ & $6(14 \%)$ & 0.7 \\
\hline Daclizumab & 0 & $16(36 \%)$ & 0.001 \\
\hline Basiliximab & $3(14 \%)$ & 0 & 0.03 \\
\hline $\begin{array}{l}\text { Number of patients on induction therapy } \\
\text { (Thymoglobulin, Daclizumab, Basiliximab) }\end{array}$ & $7(33 \%)$ & $22(50 \%)$ & 0.32 \\
\hline
\end{tabular}

Table 2. Clinical outcomes.

\begin{tabular}{|c|c|c|c|}
\hline & Tacrolimus $(\mathrm{n}=21)$ & Prograf $(n=44)$ & $p$-value \\
\hline Tacrolimus trough level $(\mu \mathrm{g} / \mathrm{L})^{*}$ & $8.8 \pm 1.8$ & $7.9 \pm 1.8$ & 0.07 \\
\hline Diabetes post-transplant & $21(100 \%)$ & $37(84 \%)$ & 0.18 \\
\hline CMV infection & 0 & $4(9 \%)$ & 0.29 \\
\hline Opportunistic infection & $1(2 \%)$ & $4(9 \%)$ & 1 \\
\hline Rejection rate 1R (mean episodes/patient-day) & $0.022 \pm 0.01$ & $0.023 \pm 0.01$ & 0.69 \\
\hline Rejection rate 2R (mean episodes/patient-day) & $0.0002 \pm 0.001$ & $0.0011 \pm 0025$ & 0.14 \\
\hline Rejection rate 3R (mean episodes/patient-day) & $0.0002 \pm 0.001$ & 0 & 0.15 \\
\hline Cardiac index 1 month post-transplant $\left(\mathrm{L} / \mathrm{min} / \mathrm{m}^{2}\right)$ & $3.0 \pm 0.7$ & $2.8 \pm 0.6$ & 0.17 \\
\hline
\end{tabular}


lular rejection was noted between the groups. There was insufficient data to permit comparison of risk of antibody-mediated rejection (AMR), as routine C3d/C4d staining as recommended by the 2009 Banff conference was only initiated at our institution shortly before generic tacrolimus was available.

The causes of death in the Prograf-treated patients were aspiration pneumonia with subsequent Staphylococcus aureus brain emboli and coronary allograft vasculopathy. The causes of deaths in the tacrolimus-treated patients were Gram-negative sepsis, andamiodaroneinduced lung toxicity with secondary Enterocoooccus and Mycobacterium avium complex (MAC) pneumonia.

\section{Discussion}

To our knowledge, this is the first systematic study on rejection rates in patients treated with generic tacrolimus versus Prograf. It is reassuring that this preliminary data does not demonstrate a significant difference in biopsyproven cellular rejection during the first 6 months posttransplant.

There are a number of limitations to this retrospective study. Most importantly, the number of tacrolimustreated patients is small $(n=21)$. Although we did not observe a difference in short-term outcomes, it is possible that a larger sample size would yield different results. The rate of death in the tacrolimus-treated group is consistent with previous reports and as both deaths were related to infection, it is unlikely that insufficient immunosuppression from tacrolimus played a role. The follow up period (6 months) in this analysis is relatively short. The rate of rejection is typically highest in this period; it would be expected that the risk of allograft rejection due to generic tacrolimus would also be highest in the first 6 months. The lack of a difference is therefore reassuring. This study was also limited to a single-center, which limits the generalizability. We do not have pharmacokinetics, as this was a retrospective study. In addition, this retrospective analysis does not address the safety of late conversion from Prograf to tacrolimus. Despite the limitations, we believe this early report to be useful and reassuring for clinicians faced with the option of initiating generic tacrolimus.

\section{Conclusion}

In this retrospective review of a "real-world" tacrolimus- based immunosuppressive regimen, we found that the occurrence of biopsy-proven acute cellular rejection at six months was comparable in patients treated with generic tacrolimus when compared to Prograf-treated patients. Although this report is limited by a small sample size, the lack of an early signal for rejection is reassuring. Larger, prospective studies are needed to clearly determine the effect of generic tacrolimus on short and longterm outcomes after heart transplantation.

\section{Acknowledgements}

We would like to thank the University of Minnesota heart transplant coordinators for their assistance with chart review.

\section{REFERENCES}

[1] J. Stehlik, L. B. Edwards, A. Y. Kucheryavaya, P. Aurora, J. D. Christie, R. Kirk, et al., "The Registry of the International Society for Heart and Lung Transplantation: Twenty-Seventh Official Adult Heart Transplant Report-2010," The Journal of Heart and Lung Transplantation, Vol. 29, No. 10, 2010, pp. 1089-1103. doi:10.1016/j.healun.2010.08.007

[2] P. A. Uber, H. J. Ross, A. O. Zuckermann, S. C. Sweet, P. A. Corris, K. McNeil, et al., "Generic Drug Immunosuppression in Thoracic Transplantation: An ISHLT Educational Advisory," The Journal of Heart and Lung Transplantation, Vol. 28, No. 7, 2009, pp. 655-660. doi:10.1016/j.healun.2009.05.001

[3] H. A. Abdulnour, C. E. Araya and V. R. Dharnidharka, "Comparison of Generic Tacrolimus and Prograf Drug Levels in a Pediatric Kidney Transplant Program: Brief Communication,” Pediatric Transplantation, Vol. 14, No. 8, 2010, pp. 1007-1011. doi:10.1111/j.1399-3046.2010.01393.x

[4] J. A. Petan, N. Undre, M. R. First, K. Saito, T. Ohara, O. Iwabe, et al., "Physiochemical Properties of Generic Formulations of Tacrolimus in Mexico," Transplantation Proceedings, Vol. 40, No. 5, 2008, pp. 1439-1442. doi:10.1016/j.transproceed.2008.03.091 